\section{Cumulation and flexibil- ity with infusions of neuromuscular blocking drugs}

François Donati PhD MD FRCPC
$\mathrm{T}$ $\mathrm{HE}$ major problem addressed in neuromuscular research over the past $60 \mathrm{yr}$ has been to provide a fast onset/rapid recovery nondepolarizing agent without side effects. The driving force behind this agenda is a patient safety problem, that is the possibility to secure the airway within 1-1.5 min and a rapid (5-10 min) recovery should intubation fail. This quest for new agents has not produced the perfect drug, but over the past decades, a trend towards faster onset, shorter duration compounds can be observed.

The availability of short acting neuromuscular blockers has another benefit quite separate from the rapid on/off property. It allows the clinician to increase or decrease rapidly the intensity of surgical relaxation, depending on actual need, and this is best accomplished when neuromuscular blocking drugs are given as infusions. The depth of anesthesia required for a given surgical procedure is not constant. The need for muscle relaxation also varies throughout surgery. It is probably highest at the time of tracheal intubation, is moderate at peritoneal incision and closure, and lowest at skin closure. These rough guidelines depend on the patient and the procedure. Infusions aside, anesthesiologists commonly inject neuromuscular relaxants according to need. A relatively large dose is given at the beginning for tracheal intubation, with no response seen at the adductor pollicis with indirect nerve stimulation. A somewhat more modest level of relaxation is needed for the start of the surgery, less relaxation is usually present for most of the procedure, and top-up doses are often needed for closure. Full recovery is a must at the end of the case.

Provided that recovery is rapid, there is, however, very little disadvantage in keeping relaxation relatively constant at a deep level throughout surgery. Most modern neuromuscular relaxants have virtually no cardio- vascular effects, so an overdose has few, if any, immediate consequences. This is not the case for narcotics, inhalational agents and intravenous anesthetics, all of which produce hypotension if a large dose is administered. Moreover, the dose of muscle relaxant can be adjusted by using a nerve stimulator. Thus, an attractive solution for the administration of muscle relaxants is by infusion, adjusted to fit most requirements during the case (usually one twitch in the train-of-four). The basic properties of the perfect neuromuscular blocking drug for administration by infusion can be imagined easily: (1) fast onset to have the option to increase relaxation quickly if required; (2) rapid recovery because neuromuscular function must return to normal after anesthesia; and (3) constant infusion rate to keep the effect constant with time. The first two of these properties are part of what is considered essential for an ideal neuromuscular blocking drug. In addition, a constant infusion rate allows profound relaxation without concern for recovery, with the anesthesiologist's attention devoted to other tasks. To accomplish this, a drug must be non-cumulative, which intuitively means that under steady-state conditions, the amount of active drug in the body remains constant. The practical consequences of a lack of cumulation is that a constant infusion of drug keeps effect constant and repeated bolus doses have the same duration of action.

Infusions of neuromuscular relaxants have been used for several decades now, but each agent has its own limitations. Succinylcholine was used frequently for short procedures as an infusion drug when all nondepolarizing drugs were long-acting. Onset and recovery are both rapid. However, the characteristics of blockade change with time. Tachyphylaxis occurs after $30-45 \mathrm{~min}$, that is the infusion rate has to be increased from a typical value of $50 \mu \mathrm{g} \cdot \mathrm{kg}^{-1} \cdot \mathrm{min}^{-1}$ to $100 \mu \mathrm{g} \cdot \mathrm{kg}^{-1} \cdot \mathrm{min}^{-1}$ to provide the same degree of

Address correspondence to: Dr. François Donati, Département d'anesthésiologie, Université de Montréal, Centre Hospitalier de l'Université de Montréal, Campus Hôtel-Dieu, 3840 rue St-Urbain, Montréal, Québec, H2W 1T8 Canada. E-mail: donatif@medclin.umontreal.ca 
blockade. ${ }^{1}$ Phase II block, which is characterized by fade following train-of-four stimulation, also appears at about the same time. Recovery from Phase II block is considerably slower than Phase I block. In addition, succinylcholine infusions are associated with the same contra-indications and side-effects as bolus doses of the drug. Thus, succinylcholine infusions have been reserved for short procedures, and in recent years, replaced by short or intermediate acting non-depolarizing drugs given as bolus or infusions.

With the almost simultaneous introduction of atracurium and vecuronium in the 1980's, the presence of relatively rapid recovery made infusions practical. The pharmacokinetic characteristics of these agents are not, however, identical, and this led to different predictions with respect to their behaviour when administered as infusions. Termination of action of atracurium depends on Hofmann elimination and ester hydrolysis, and these processes occur at a constant rate irrespective of plasma concentration and past history of drug administration. Thus, it was predicted that atracurium, if given at a constant rate, would produce constant blockade with time. Also, giving top-up doses of equal size would produce the same duration of relaxation, and this duration would not change with time. These predictions have been confirmed by clinical evidence by Martineau et $a l^{2}$ in patients not given potent inhalational vapours. The infusion rate to keep blockade constant (approximately $5 \mu \mathrm{g} \cdot \mathrm{kg}^{-1} \cdot \mathrm{min}^{-1}$ ) did not vary with time, which suggests that atracurium does not cumulate. In another study with incremental doses, atracurium was also found to have the same duration after each bolus, which also suggests lack of cumulation. ${ }^{3}$ However, in a group of subjects receiving atracurium by infusion, the same authors found that the infusion rate had to be decreased after the first 30 min or so to keep blockade constant.

The situation is different for vecuronium, which relies on redistribution, not on metabolism, for termination of effect. This is similar to the case of thiopental where duration of action depends on redistribution to fatty tissue. If vecuronium or thiopental is given by infusion, it is expected that the redistribution process will become less and less efficient as redistribution organs become more and more saturated. As a result, the infusion rate required for constant effect is expected to decrease with time, as was found in several studies. ${ }^{2-3}$ For example, in Martineau et al. ${ }^{2}$ the infusion requirements of vecuronium decreased by almost half over a two-hour period, from 1 to $0.5 \mu \mathrm{g} \cdot \mathrm{kg}^{-1} \cdot \mathrm{min}^{-1}$. Redistribution can also be predicted to have two more consequences. If the drug is given by incremental topup doses, the duration of each dose is expected to increase with time, and upon termination of anesthesia and surgery, recovery is expected to take longer after prolonged administration. ${ }^{3-5}$ In practice, howev$\mathrm{er}$, the increase in vecuronium duration of action of successive top-up doses is small, and recovery is rapid if only moderate blockade $(<90 \%)$ is maintained. The most likely explanation for this observation is through the concept of context-sensitive half-time. Although the idea has been developed for narcotics and intravenous anesthestics, there is no reason not to apply it to neuromuscular blocking drugs. In this setting, vecuronium can be modeled as having two peripheral compartments which fill up progressively as the drug is given by infusion or repeated bolus. Upon termination of the infusion or after the last bolus dose, the plasma levels (central compartment) are initially governed by exit rate from the central compartment, because the concentration gradients between central and peripheral compartments are too small to refill the central compartment significantly. Thus, initial recovery after an infusion is relatively rapid. However, as plasma concentrations decrease, a large concentration gradient between peripheral and central compartments is now established, which fills the central compartment as it empties, and this slows down the rate of decrease of plasma concentration. Taken together, the concept of context sensitive half-time and experimental evidence suggest that cumulation occurs with vecuronium, but the phenomenon is limited if only moderate levels of blockade are achieved by intermittent bolus or infusion, and in the case of intermittent dosing, only small incremental doses are given.

The next advance in infusions was provided with the introduction of mivacurium. This drug had a better profile as an infusion drug than atracurium and vecuronium because its recovery rate was more rapid, thus allowing for faster changes towards less relaxation. Mivacurium is a mixture of three isomers, cis-trans, trans-trans and cis-cis. The first two are metabolized rapidly by plasma cholinesterase and the cis-cis isomer, which makes up only $6 \%$ of the commercial preparation, seems to be sufficiently inactive not to alter the rate of recovery even after prolonged infusions. ${ }^{6}$ Mivacurium is non-cumulative, and in the absence of potent inhalational anesthetics, its infusion rate for constant blockade (typically $5-7 \mu \mathrm{g} \cdot \mathrm{kg}^{-1} \cdot \mathrm{min}^{-1}$ ) remains constant over many hours. ${ }^{7}$ The usual method of administration of mivacurium when an infusion is planned is to give a bolus of $0.2-0.25 \mathrm{mg} \cdot \mathrm{kg}^{-1}$ to facilitate tracheal intubation and start the infusion when some evidence of recovery is manifest, that is $\mathbf{1 5 - 2 0} \mathrm{min}$ later. At that time, its should be noted that the infusion rate required to keep $90-95 \%$ blockade is high, ${ }^{7,8}$ because enough 
drug has to be provided not only to maintain blockade, but also to prevent it from wearing off. Among currently available drugs, it appears that mivacurium is best suited for administration by infusion. It has rapid recovery and constant effect with time after the infusion rate has been stabilized. Only the relatively slow onset poses any problem.

Perhaps because of mivacurium's greater potential in this regard, rocuronium and cisatracurium have been given less attention as infusion drugs. The pharmacokinetic properties of these newer agents suggested that they would behave much like vecuronium and atracurium, respectively. In this issue of the Journal, Miller $e t$ al. ${ }^{9}$ measured the infusion rate required for both drugs, to determine if the drug requirement changed with time. It came as no surprise to observe that over a two hour period, the infusion rate of rocuronium had to be decreased from 10 to 5 $\mu \mathrm{g} \cdot \mathrm{kg}^{-1} \cdot \mathrm{min}^{-1}$, and this was attributed to the drug's redistribution. Surprisingly, however, cisatracurium presented the same behavior. The infusion rate required to keep blockade constant decreased with time, from 1.7 to $0.9 \mu \mathrm{g} \cdot \mathrm{kg}^{-1} \cdot \mathrm{min}^{-1}$. This was clearly unexpected because cistracurium, like atracurium, is not redistributed and undergoes Hofmann elimination at a constant rate. Before jumping to explanations, the design of the study should be compared with that of the previous investigation, ${ }^{2}$ performed by the same group, where atracurium and vecuronium were compared. The first obvious difference was the background anesthetic, which included propofol in the recent work, and was limited to nitrous oxide-narcotic in the previous paper. A time-dependent potentiation of neuromuscular blockade by propofol is unlikely, and could not explain why rocuronium and cisatracurium presented the same behaviour. The other difference has to do with the size of the bolus dose given. In the atracurium-vecuronium study, ${ }^{2}$ the first dose was close to the $\mathrm{ED}_{95}$ of each drug, and the infusion was started almost immediately, before any recovery was apparent. In the present study, the bolus dose was equivalent to $2 \times \mathrm{ED}_{95}$ and the infusion not begun until at least $5 \%$ recovery was observed. At that time, the infusion rate had to large enough not only to maintain relaxation, but also to prevent recovery from taking place. If this hypothesis is accepted, then the similarity between cisatracurium and rocuronium suggests that both have the same cumulation characteristics. In fact, rocuronium exhibits very little cumulation, at least in young adults receiving bolus doses. ${ }^{10}$ Previous studies suggest a decrease in infusion rate with time for rocuronium, ${ }^{11}$ but for cisatracurium as well. ${ }^{12,13}$
The newer agent rapacuronium has two of the characteristics of a good infusion drug. Both onset and offset are rapid. However, rapacuronium is metabolized into ORG 9488, which has twice the potency of the parent compound and is longer acting. ${ }^{14}$ After a one-hour infusion, recovery rate is slow er than after a bolus dose. ${ }^{15}$ Mean infusion rates are 55 $\mu \mathrm{g} \cdot \mathrm{kg}^{-1} \cdot \mathrm{min}^{-1}$, but only $41 \mu \mathrm{g} \cdot \mathrm{kg}^{-1} \cdot \mathrm{min}^{-1}$ at the end of the infusion period. Longer infusions have not been tried, but it is predicted that recovery would be even more prolonged. Considering that even non-cumulative drugs such as atracurium and cisatracurium may present variations in their infusion rates, however, final recommendations should not be made until the data are available.

The study by Miller et al..$^{9}$ and the available literature ${ }^{3,5,7,8,11,12,13}$ suggest that atracurium, vecuronium, mivacurium, cisatracurium and rocuronium behave similarly when an infusion is given after an intubating dose. The infusion rate is greater during the transition from bolus dose to an infusion regimen than during steady state infusion, even for so-called non-cumulative agents. How can these data be applied to the more typical situation when requirements for relaxation are varying? Compared with intravenous anesthetics like propofol or narcotics like remifentanil, muscle relaxants have a slow onset and offset, so any step change in the infusion rate will take many minutes before steady state is achieved. Considering that the transition from bolus to infusion requires many dose adjustments and time (typically $30 \mathrm{~min}$ ), it does not appear practical to change the infusion rate depending on the varying requirements during the surgical procedure. This slow response implies that except for very long cases, infusions are best used when adjusted to keep relaxation constant, at a level deep enough to cover all needs for the procedure.

\section{Accumulation et flexi- bilité avec des perfu- sions de curares}

Le problème de curarisation qui occupe les esprits depuis 60 ans est celui de trouver un agent nondépolarisant avec une action rapide et courte sans effets secondaires. Cette recherche est motivée par un problème de sécurité du patient, soit la possibilité d'intuber en 
$1-1,5$ min et d'obtenir une récupération rapidement (5 à $10 \mathrm{~min}$ ) en cas d'échec. On n'a pas encore trouvé la molécule parfaite, mais depuis quelques décennies, on peut observer une tendance vers des produits à action plus rapide et de durée plus courte.

La présence de curares à durée d'action courte est avantageuse sous un autre aspect. Elle permet à l'anesthésiologiste d'augmenter ou de diminuer rapidement au besoin l'intensité de la curarisation, en administrant de préférence les curares en perfusion. La profondeur de l'anesthésie nécessaire pour une intervention chirurgicale typique varie en fonction du temps. Il existe aussi des variations du niveau approprié de curarisation : élevé pour l'intubation trachéale, intermédiaire pour l'incision et la fermeture du péritoine et bas pour la fermeture de la peau, en modulant selon le patient et l'intervention. Si l'on fait abstraction des perfusions, les anesthésiologistes administrent les curares selon les besoins du malade. On donne habituellement une forte dose au début pour l'intubation trachéale, assez pour produire l'abolition de la réponse au niveau de l'adducteur du pouce à la suite de la stimulation du nerf cubital. On a besoin d'un peu moins de curarisation pour le début de l'opération, encore moins pour la majeure partie de l'intervention, quitte à recurariser vers la fin. On doit obtenir une récupération complète avant le réveil.

Toutefois, pourvu que la récupération s'effectue rapidement, il y a peu d'inconvénients à garder le niveau de curarisation à peu près constant pendant toute l'opération. La plupart des curares de dernière génération sont pratiquement dépourvus d'effets cardiovasculaires, de sorte qu'une surdose a peu ou pas de conséquences immédiates. Ce n'est pas le cas des morphiniques, des halogénés ou des anesthésiques intraveineux, qui produisent tous une hypotension à forte dose. De plus, la dose de curare peut être ajustée en fonction de la réponse à la stimulation nerveuse. Ainsi, une façon élégante d'administrer les curares est de donner une perfusion dont la vitesse est ajustée pour répondre à la majorité des besoins prévus (habituellement une réponse à la suite de la stimulation en train-de-quatre). On peut imaginer facilement les caractéristiques essentielles d'un curare idéal pour l'administration en perfusion : (1) un début d'action rapide, pour pouvoir augmenter la curarisation au besoin; (2) une récupération rapide pour que la force musculaire retourne à la normale à la fin de l'anesthésie et (3) une vitesse de perfusion constante correspondant à une curarisation constante en fonction du temps. Les deux premières caractéristiques sont considérées essentielles pour un curare parfait. De plus, une vitesse de perfusion constante, pour produire une curarisation intense sans se soucier de la récupération, permet à l'anesthésio- logiste de porter attention à d'autres tâches. Pour ce faire, un médicament doit ne pas produire d'accumulation, ce qui, intuitivement, veut dire qu'en régime stable, la quantité de produit actif présent dans l'organisme demeure constante. Pratiquement, l'absence d'accumulation implique qu'une perfusion à vitesse constante garde l'effet constant et que des injections répétées ont toutes une durée d'action identique.

On utilise des perfusions de curare depuis plusieurs décennies mais chaque médicament a ses propres limites. La succinylcholine était populaire pour des interventions courtes quand les seuls curares non dépolarisants disponibles étaient de longue durée. Le début d'action et la récupération sont tous deux rapides. Toutefois, les caractéristiques du bloc changent en fonction du temps. Une tachyphylaxie, soit une augmentation de la vitesse d'infusion requise de 50 à 100 $\mu \mathrm{g} \cdot \mathrm{kg}^{-1} \cdot \mathrm{min}^{-1}$ pour maintenir la curarisation constante, s'installe après 30 à $45 \mathrm{~min} .{ }^{1}{ }^{1}$ Ceci coïncide avec l'apparition d'un bloc de phase II, où l'on observe un épuisement à la suite d'une stimulation en train-dequatre. La récupération après un bloc de phase II est plus longue qu'après un bloc de Phase I. De plus, la succinylcholine possède les mêmes contre indications et les mêmes effets secondaires en perfusion qu'en dose unique. Ainsi, les perfusions de succinylcholine ont été utilisées surtout pour les interventions de courte durée. Récemment, elles ont été remplacées par des curares non dépolarisants en injection ou en perfusion.

Lorsque l'atracurium et le vécuronium sont devenus disponibles presque en même temps dans les années 1980, leur récupération assez rapide a permis leur utilisation en perfusion. Toutefois, la pharmacocinétique de ces deux molécules est différente, ce qui permet de prédire des caractéristiques de perfusion différentes. L'effet de l'atracurium se dissipe sous l'action combinée de l'élimination de Hofmann et de l'hydrolyse du lien ester, à un taux constant, peu importe la concentration plasmatique ou l'historique de l'administration. Ainsi, on en déduit que l'atracurium donné en perfusion à vitesse constante produira un bloc qui ne changera pas avec le temps. On prédit aussi que l'effet produit par l'administration successive de doses identiques sera de durée égale. Martineau et coll. ${ }^{2}$ ont vérifié ces prédictions en clinique chez des patients qui ne recevaient pas d'halogénés. La vitesse de perfusion (environ $5 \mu \mathrm{g} \cdot \mathrm{kg}^{-1} \cdot \mathrm{min}^{-1}$ ) qui correspondait à un bloc constant ne variait pas en fonction du temps, ce qui indique que l'atracurium ne s'accumule pas. Dans une autre étude où l'on a donné l'atracurium en injections répétées, on a aussi trouvé des durées d'action égales après chaque dose, donc une 
absence probable d'accumulation. ${ }^{3}$ Les auteurs de cette étude ont aussi inclus un groupe de sujets chez qui l'atracurium était administré par perfusion, et pour ces patients, la vitesse de perfusion devait toutefois être diminuée après une trentaine de minutes pour garder le bloc constant.

La situation est différente pour le vécuronium, dont l'effet se dissipe non pas par métabolisme, mais à cause d'une redistribution. Le cas du thiopental, qui doit sa courte durée d'action à une redistribution vers les tissus adipeux, est semblable. Lors d'une perfusion de vécuronium ou de thiopental, on s'attend à ce que la redistribution soit de moins en moins efficace à mesure que les organes vers lesquels il y a redistribution deviennent saturés. Pour garder un effet constant, on aura besoin d'une perfusion dont la vitesse diminue avec le temps, comme plusieurs études l'on démontré. ${ }^{2,3}$ Par exemple, d'après Martineau et coll. ${ }^{2}$ les besoins en vécuronium diminuent de moitié environ sur une période de deux heures, de 1 à $0,5 \mu \mathrm{g} \cdot \mathrm{kg}^{-1} \cdot \mathrm{min}^{-1}$. On peut prédire deux autres conséquences de l'effet de redistribution. Pour une série de doses égales, la durée d'action de chacune devrait s'allonger avec le temps et la récupération après l'anesthésie et l'intervention chirurgicale devrait être plus lente si l'intervention est longue. ${ }^{3-5}$ Toutefois, en pratique, la durée d'action du vécuronium après des doses répétées augmente peu avec le temps et la récupération demeure rapide si l'on se contente d'un bloc modéré (<90\%). L'explication la plus probable de ce phénomène passe par l'idée de la demi-vie contextuelle. ${ }^{6}$ Le concept a été mis au point pour les morphiniques et les anesthésiques intraveineux, mais il n'y a aucune raison de ne pas l'appliquer aux curares. Le vécuronium peut être considéré comme ayant deux compartiments périphériques qui se remplissent à mesure que le médicament est administré soit en perfusion, soit en doses répétées. Lorsque l'on cesse la perfusion ou après la dernière dose injectée, les concentrations plasmatiques (compartiment central) diminuent d'abord en fonction du taux de sortie du compartiment central, parce que les gradients de concentration entre les compartiments périphériques et le compartiment central sont trop faibles pour que les premiers se déversent dans le second de façon significative. Toutefois, à mesure que les concentrations plasmatiques diminuent, ce gradient de concentration augmente, ce qui remplit le compartiment central à partir des compartiments périphériques, diminuant d'autant la vitesse à laquelle les concentrations plasmatiques baissent. Le concept de demi-vie contextuelle explique bien les données expérimentales en prédisant la présence d'accumulation pour le vécuronium, mais ce phénomène est de portée limitée si les niveaux de curarisation atteints par perfusion ou doses répétées sont modérés et si les doses répétées sont modestes.

Avec son temps de récupération plus court que celui de l'atracurium et du vécuronium, l'arrivée du mivacurium a été saluée comme un progrès pour l'administration en perfusion, parce qu'une diminution plus rapide du relâchement musculaire était possible. Le mivacurium est constitué de trois isomères, cis-trans, trans-trans et cis-cis. Les deux premiers sont métabolisés rapidement par la cholinestérase plasmatique et l'isomère cis-cis, qui ne représente que $6 \%$ de la préparation disponible commercialement, semble être suffisamment inactif pour que la vitesse de récupération demeure la même peu importe la durée de la perfusion. ${ }^{6}$ Le mivacurium ne s'accumule pas et si l'on évite les halogénés, la vitesse de perfusion nécessaire pour garder un bloc constant (ordinairement 5-7 $\mu \mathrm{g} \cdot \mathrm{kg}^{-1} \cdot \mathrm{min}^{-1}$ ) reste stable pendant plusieurs heures. ${ }^{7}$ La façon habituelle d'administrer le mivacurium lorsque l'on prévoit une perfusion est de donner une dose de 0,2 à $0,25 \mathrm{mg} \cdot \mathrm{kg}^{-1}$ avant l'intubation trachéale et de commencer la perfusion 15-20 min plus tard, à la première manifestation de signes de récupération. Il est à noter qu'à ce moment, la vitesse de perfusion utile pour garder un bloc de 90 à $95 \%$ est normalement assez élevée, ${ }^{7,8}$ parce qu'il faut assez de produit non seulement pour maintenir la curarisation, mais aussi pour empêcher qu'elle se dissipe. Parmi les curares disponibles actuellement, le mivacurium est le mieux adapté à l'administration en perfusion. Il a une récupération rapide et un effet constant une fois la vitesse de perfusion stabilisée. Son seul handicap est son délai d'action lent.

Sans doute à cause du meilleur potentiel du mivacurium, on a peu étudié les perfusions de rocuronium et de cisatracurium. En se basant sur leurs propriétés pharmacocinétiques, on prévoyait que ces curares se comporteraient respectivement comme le vécuronium et l'atracurium. Dans ce numéro du Journal, Miller et coll. ${ }^{9}$ ont mesuré, pour chacun de ces curares, la vitesse de perfusion appropriée et ont cherché à savoir si cette vitesse changeait avec le temps. On ne s'est pas surpris du fait que la vitesse de perfusion du rocuronium devait être diminuée de 10 à $5 \mu \mathrm{g} \cdot \mathrm{kg}^{-1} \cdot \mathrm{min}^{-1}$ au cours d'une période de deux heures. On a attribué ce phénomène à la redistribution du rocuronium. Toutefois, le cisatracurium s'est comporté de façon semblable, avec des vitesses de perfusion passant de 1,7 à $0,9 \mu \mathrm{g} \cdot \mathrm{kg}^{-1} \cdot \mathrm{min}^{-1}$. Ceci était inattendu, puisque le cisatracurium, tout comme l'atracurium, est éliminé à un taux constant par la réaction d'Hofmann. Avant de sauter aux conclusions, il convient de bien comparer la dernière étude avec celle où l'on administrait du 
vécuronium et de l'atracurium et qui a été réalisée par la même équipe. ${ }^{2}$ La première différence évidente est la technique anesthésique de base, qui incluait du propofol dans l'étude la plus récente et qui ne renfermait que du protoxyde d'azote et un morphinique dans l'article précédent. Il est peu probable que le propofol potentialise le bloc neuromusculaire d'un façon qui s'intensifie avec temps et ceci n'expliquerait pas pourquoi le rocuronium et le cisatracurium auraient le même comportement. L'autre différence est dans la dose de charge. Dans l'étude sur l'atracurium et le vécuronium, ${ }^{2}$ cette dose était près de la $\mathrm{DA}_{5}$ (dose active), et la perfusion était mise en route presque immédiatement, avant que l'on observe une récupération. Dans l'étude actuelle, la dose de charge s'établissait à $2 \mathrm{x} \mathrm{DA}_{95}$ et la perfusion n'était commencée que lorsque la récupération atteignait $5 \%$. Dans ce dernier cas, il fallait une vitesse de perfusion assez grande non seulement pour garder le bloc constant, mais aussi pour empêcher que la récupération progresse. Si l'on accepte cette hypothèse, on doit conclure que le cistracurium et le rocuronium ont tous deux les mêmes caractéristiques d'accumulation. D'ailleurs, le rocuronium s'accumule peu, du moins chez les jeunes adultes recevant des doses répétées. ${ }^{10}$ D'autres études ont décrit une diminution de la vitesse de perfusion fonction du temps pour le rocuronium, ${ }^{11}$ mais aussi pour le cisatracurium. ${ }^{12,13}$

Le rapacuronium, le dernier-né des curares, possède deux des caractéristiques recherchées pour une perfusion. Le délai d'action et la récupération sont tous deux rapides. Toutefois, le rapacuronium est métabolisé en ORG 9488, deux fois plus puissant et agissant plus longtemps que la molécule-mère. ${ }^{14}$ Après une perfusion d'une heure, la récupération est plus lente qu'après une dose unique. ${ }^{15}$ Les vitesses de perfusion moyennes se chiffrent à $55 \mu \mathrm{g} \cdot \mathrm{kg}^{-1} \cdot \mathrm{min}^{-1}$, mais à seulement $41 \mu \mathrm{g} \cdot \mathrm{kg}^{-1} \cdot \mathrm{min}^{-1}$ après une heure. On n'a pas essayé de perfusions plus longues, mais on prévoit que la récupération serait encore plus lente. Néanmoins, sachant que des produits peu enclins à l'accumulation comme l'atracurium et le cisatracurium présentent des variations dans la vitesse de perfusion, il faudrait se garder de formuler des recommandations finales avant que les données soient disponibles.

L'étude de Miller et coll. ${ }^{9}$ et d'autres articles publiés $3,5,7,8,11,12,13$ suggèrent que l'atracurium, le vécuronium, le mivacurium, le cisatracurium et le rocuronium possèdent tous des caractéristiques semblables lorsque donnés en perfusion après une dose d'intubation. La vitesse de perfusion est plus grande pendant la transition entre la dose de charge et la perfusion, et ceci s'applique même pour les produits réputés pour leur absence d'accumulation. Comment pouvons-nous appliquer ces résultats au cas plus typique où les besoins en curarisation varient? Les curares ont un début d'action et une récupération plus lentes que les anesthésiques intraveineux comme le propofol et les morphiniques comme le rémifentanil, de sorte qu'il faut attendre plusieurs minutes avant qu'un changement de vitesse de perfusion produise un nouvel état stable. Pour établir une perfusion après une dose de charge, il faut plusieurs ajustements et un certain temps (de l'ordre de $30 \mathrm{~min}$ ). Ainsi, il semble peu pratique de changer la vitesse de perfusion pour s'ajuster aux changements dans les besoins en curarisation en cours d'intervention. Cette réponse lente fait que, mis à part les cas très longs, il vaut mieux viser un niveau de curarisation constant et le choisir suffisamment profond pour répondre à tous les besoins durant l'opération.

\section{References}

1 Donati F, Bevan DR. Long-term succinylcholine infusion during isoflurane anesthesia. Anesthesiology 1983; 58: 6-10.

2 Martineau RJ, St.-Jean B, Kitts JB, et al. Cumulation and reversal with prolonged infusions of atracurium and vecuronium. Can J Anaesth 1992; 39: 670-6.

3 Diefenbach C, Mellinghoff $H$, Grond S, Buzello W. Atracurium and vecuronium: repeated bolus injection versus infusion. Anesth Analg 1992; 74: 519-22.

4 Fisher DM, Rosen JI. A pharmacokinetic explanation for increasing recovery time following larger or repeated doses of nondepolarizing muscle relaxants. Anesthesiology 1986; 65: 285-91.

5 McCoy EP, Mirakhur RK, Maddineni VR, Loan PB, Connolly F. Administration of rocuronium (Org 9426) by continuous infusion and its reversibility with anticholinesterases. Anaesthesia 1994; 49: 940-5.

6 Lien CA, Schmith VD, Embree PB, Belmont MR, Wargin WA, Savarese JJ. The pharmacokinetics and pharmacodynamics of the stereoisomers of mivacurium in patients receiving nitrous oxide/opioid/barbiturate anesthesia. Anesthesiology 1994; 80: 1296-302.

7 Goudsouzian N, Chakravorti S, Denman W, Schwartz A, Yang HS, Cook DR. Prolonged mivacurium infusion in young and elderly adults. Can J Anaesth 1997; 44: 955-62.

8 Diefenbach C, Mellinghoff $H$, Lynch J, Buzello W. Mivacurium: dose-response relationship and administration by repeated injection or infusion. Anesth Analg 1992; 74: 420-3.

9 Miller DR, Wherrett C, Hull K, Watson J, Legault S. Cisatracurium vs rocuronium: Cumulation characteristics during continuous infusion. Can J Anesth 2000; 47: 943-49. 
10 Bevan DR, Fiset P, Balendran P, Law-Min JC, Ratcliffe $A$, Donati F. Pharmacodynamic behaviour of rocuronium in the elderly. Can J Anaesth 1993; 40: 127-32.

11 Shanks CA, Fragen RJ, Ling D. Continuous intravenous infusion of rocuronium (ORG 9426) in patients receiving balanced, enflurane, or isoflurane anesthesia. Anesthesiology 1993; 78: 649-51.

12 Belmont MR, Lien CA, Quessy S, et al. The clinical neuromuscular pharmacology of 51W89 in patients receiving nitrous oxide/opioid/barbiturate anesthesia. Anesthesiology 1995; 82: 1139-45.

13 Mellinghoff $H$, Radbruch L, Diefenbach C, Buzello W. A comparison of cisatracurium and atracurium: onset of neuromuscular block after bolus injection and recovery after subsequent infusion. Anesth Analg 1996; 83:

1072-5.

14 Schiere S, Proost JH, Schuringa M, Wierda JM.

Pharmacokinetics and pharmacokinetic-dynamic relationship between rapacuronium (Org 9487) and its 3desacetyl metabolite (Org 9488). Anesth Analg 1999; 88: 640-7.

15 van den Broek L, Wierda JM, Smeulers NJ, Proost JH. Pharmacodynamics and pharmacokinetics of an infusion of Org 9487, a new short-acting steroidal neuromuscular blocking agent. Br J Anaesth 1994; 73: 331-5. 